\title{
Publisher Correction: Systemic AAV vectors for widespread and targeted gene delivery in rodents
}

Rosemary C. Challis, Sripriya Ravindra Kumar, Ken Y. Chan, Collin Challis, Keith Beadle, Min J. Jang, Hyun Min Kim, Pradeep S. Rajendran (D), John D. Tompkins, Kalyanam Shivkumar, Benjamin E. Deverman, Viviana Gradinaru (DD

Correction to: Nature Protocols https://doi.org/10.1038/s41596-018-0097-3, published online 9 January 2019

During the production process, the authors of this paper supplied revised versions of Figs. 2-5, Supplementary Tables 1-4, and Supplementary Videos 1-3, but because of publisher error, these revised items were not included in the final published version of the protocol. The figures have been updated in the PDF and HTML versions of the paper, and the revised Supplementary Information files are now available online. We note that the figures have been revised to improve their resolution only; the content of the figures and the data reflected remain unchanged. Also, print requirements impose some limits on figure resolution, but the authors have made very high-resolution versions of Figs. 2-5 available as Source data.

Published online: 16 July 2019

https://doi.org/10.1038/s41596-019-0155-5

\section{Publisher Correction: Application and optimization of CRISPR-Cas9-mediated genome engineering in axolotl (Ambystoma mexicanum)}

Ji-Feng Fei D, Wilson Pak-Kin Lou, Dunja Knapp (D), Prayag Murawala (D), Tobias Gerber, Yuka Taniguchi, Sergej Nowoshilow D, Shahryar Khattak, Elly M. Tanaka (D)

Correction to: Nature Protocols https://doi.org/10.1038/s41596-018-0071-0, published online 14 November 2018

In the version of this protocol originally published, the recipe for CAS9 buffer was incorrectly identified as a recipe for sodium acetate solution, and vice versa. These errors have been corrected in the PDF and HTML versions of the paper.

Published online: 29 January 2019

https://doi.org/10.1038/s41596-019-0138-6

\section{Publisher Correction: Sequencing cell-type-specific transcriptomes with SLAM-ITseq}

Wayo Matsushima (D), Veronika A. Herzog, Tobias Neumann (D), Katharina Gapp, Johannes Zuber (D), Stefan L. Ameres, Eric A. Miska (D)

Correction to: Nature Protocols https://doi.org/10.1038/s41596-019-0179-x, published online 26 June 2019

In the version of this paper originally published, step 10 in Box 1 incorrectly stated that the user should carry out 40 cycles of each of three temperature and time stages in the PCR run. The run should actually contain only two steps, with the second including a shift from $95{ }^{\circ} \mathrm{C}$ to $60{ }^{\circ} \mathrm{C}$. The instructions have been corrected in the PDF and HTML versions of the protocol.

Published online: 11 July 2019

https://doi.org/10.1038/s41596-019-0224-9 